\title{
Correlation Microanalysis of Localized Molecular Structure and Nano- mechanical Property of PVDF Based Copolymer
}

\author{
Wen Qian, ${ }^{*}$ Shuo Sun, ${ }^{2,3}$ Charles Nguyen, ${ }^{1}$ Stephen Ducharme, ${ }^{2}$ Joseph A. Turner ${ }^{1}$ \\ ${ }^{1}$ Dept of Mechanical \& Materials Engineering, University of Nebraska-Lincoln, Lincoln, NE 68588 \\ ${ }^{2}$ Dept of Physics \& Astronomy and Nebraska Center for Materials and Nanoscience, University of \\ Nebraska-Lincoln, Lincoln, NE 68588 \\ 3 Shanghai Institute of Technical Physics, Chinese Academy of Sciences, Shanghai, 200083, China \\ Corresponding author:wqian2@unl.edu
}

Poly (vinylidenefluoride) (PVDF) and its copolymers, are well-recognized electroactive polymers. The PVDF polymer can crystallize in a quasi-hexagonal close-packed " $\beta$-phase" structure with the dipoles of all chains aligned with maximum spontaneous polarization [1-3]. Due to the limited availability of quantitative methods for nanoscale molecular structure and mechanical analysis, the polymer organization has not been fully optimized. For such ferroelectric polymers, the influence of nanoscale molecular structure on mechanical response is not well understood. In this paper, we demonstrate nanoscale characterization and measurement techniques, by comprehensive integrating atomic force microscopy (AFM), X-ray diffraction (XRD), nano-infrared (nanoIR) spectroscopy, nanoindentation, chemical nanoIR mapping, and force mapping, to probe the localized morphology, molecular structure, molecular orientation, and mechanical stiffness distribution at the nanoscale.

The thin P(VDF-TrFE) copolymer film (80-100 nm in thickness) studied here was made of $70 \%$ vinylidine fluoride and 30\% trifluoroethylene via spin-coating. Fig. 1a shows an AFM topography image of this film with a scan area of $3 \mu \mathrm{m} \times 3 \mu \mathrm{m}$. The thin film has uniform morphology with a highly crystalline rice-grain-shaped crystals $(250 \mathrm{~nm} * 50 \mathrm{~nm})$. The XRD pattern (Fig. 1b) shows that this film is in the polar electroactive $\beta$-phase, the reflection peak of $2 \theta$ at $19.7^{\circ}$ corresponds with the (110) and (200) planes. By positioning the AFM probe tip on a single rice grain region (marked with a blue oval), we can acquire the localized IR spectrum (Fig. 1c), which clearly shows there are three fingerprints for the $\beta$-phase in the IR spectrum at $1071 \mathrm{~cm}^{-1}, 1176 \mathrm{~cm}^{-1}$, and $1428 \mathrm{~cm}^{-1}$. These represent the $\mathrm{C}-\mathrm{C}$ antisymmetric stretching, $\mathrm{CF} 2$ antisymmetric stretching, and $\mathrm{CH} 2$ bending stretch of the alltrans (TT) structure, respectively. Due to instrumental variation, the peak positions have a shift of several wavenumbers. Fig. 1d-f shows the corresponding chemical mapping of the film at fixed wavenumbers of $1084 \mathrm{~cm}^{-1}, 1181 \mathrm{~cm}^{-1}$, and $1434 \mathrm{~cm}^{-1}$ respectively. Note the distribution of these three absorption bands in the same rice grain region (marked with a red oval). Furthermore, we can identify the molecular orientation of substructure within a nanocrystal, to investigate the associated changes of localized nanoIR spectra and chemical maps by changing the input polarization of the IR light source.

Fig. 2a shows the AFM topography image of the film for a scan area of $5 \mu \mathrm{m} x 5 \mu \mathrm{m}$. Fig. $2 \mathrm{~b}$ shows the corresponding high resolution force map (140 pt by $140 \mathrm{pt})$, which was acquired by recording 19600 force curves onto the scan area. By fitting these load-displacement curves using the DerjaguinMuller-Toporov (DMT) model, the histogram shows the measured local elastic modulus primarily within the range of 1-2 Gpa (Fig. 2c). In addition, we also performed the single static indentation on bulk P(VDF-TrFE) thin film (10 $\mu$ m thickness), the measured modulus is $2.936 \mathrm{GPa}$. The integration of XRD, AFM, localized IR spectra, chemical map, nanoindentation, and force map can enable us to elucidate the relationship between localized molecular structure and mechanical property analysis, which will guide the design and fabrication of high-performance, flexible, energy-harvesting devices. 
References

[1] P. Martins et al., Progress in Polymer Science, 39 (2014) p. 683

[2] M. Kobayashi et al., Macromolecules, 8 (1975) p.158

[3] W. Qian et al. Nanotechnology, 29 (2018) p. 335702

[4] Characterization analysis was performed at the NanoEngineering Research Core Facility (NERCF). This research were supported by Nebraska Center for Energy Science Research, National Key R\&D Program of China (Grant No. 2016YFB0400801), Natural Science Foundation of China (Grant No. 61404147)

a

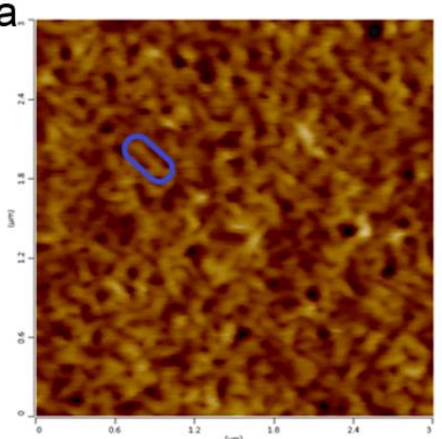

d

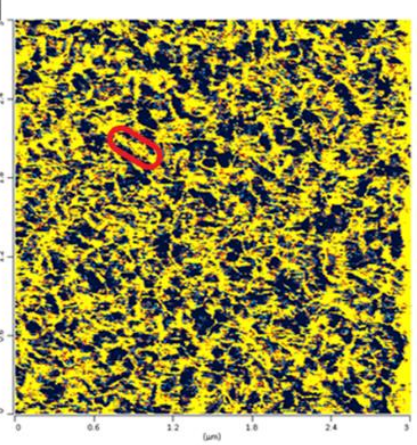

b
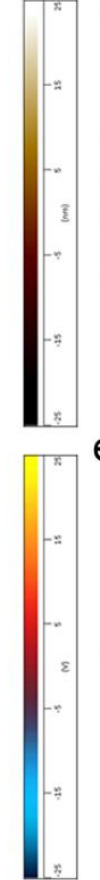

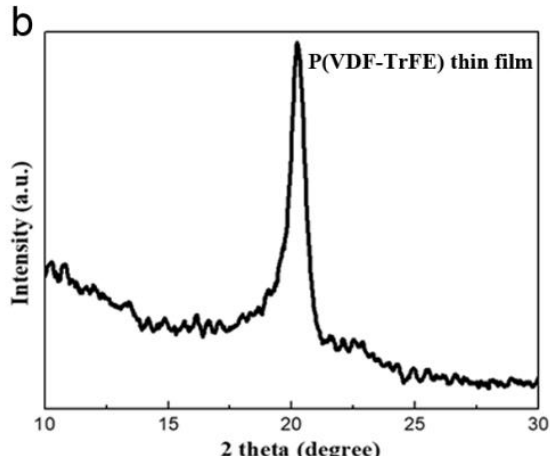

e.

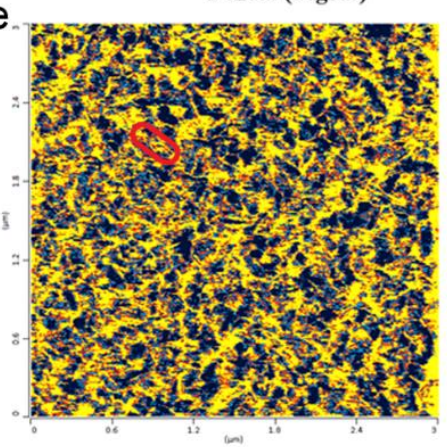

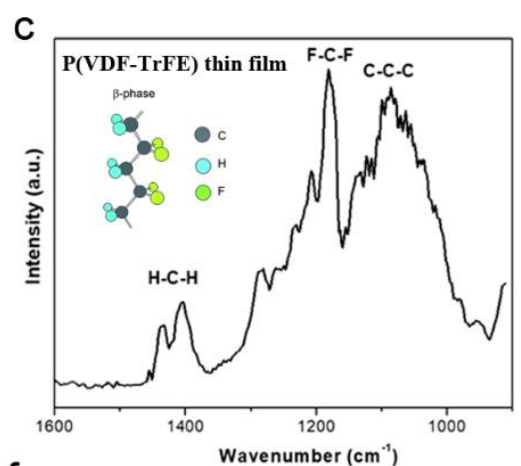

f.

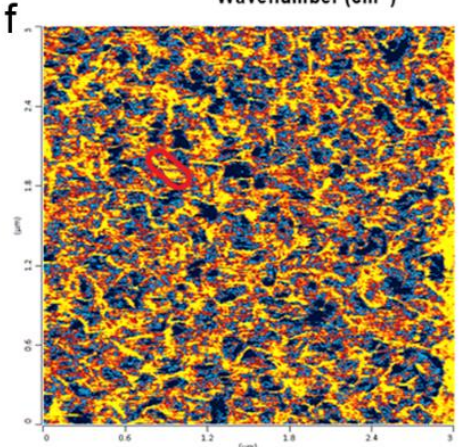

Fig. 1 (a) AFM topography image of P(VDF-TrFE) with a scan area of $3 \mu \mathrm{m}$ x $3 \mu \mathrm{m}$; (b) XRD pattern of P(VDF-TrFE) thin film; (c) Localized nanoscale IR spectra at a single rice grain region marked in Fig. 1a; (d-f) Corresponding chemical mapping image at the fixed wavenumbers of $1084 \mathrm{~cm}^{-1}, 1181$ $\mathrm{cm}^{-1}$, and $1434 \mathrm{~cm}^{-1}$ respectively, showing the map for the C-C-C antisymmetric stretching, F-C-F asymmetric stretching, and $\mathrm{H}-\mathrm{C}-\mathrm{H}$ bending absorption band.
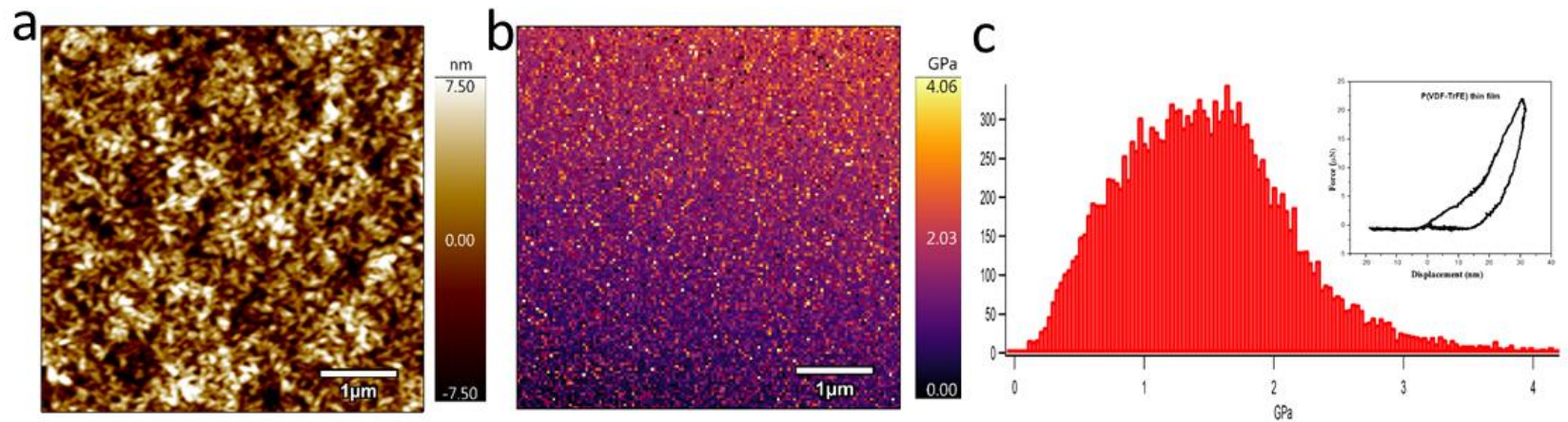

Fig. 2 (a) AFM topography image of P(VDF-TrFE) thin film with a scan area of $5 \mu \mathrm{m} \times 5 \mu \mathrm{m}$; (b) Corresponding high resolution force map; (c) Histograms of modulus value primarily within 1 2 Gpa; Static nanoindentation on a bulk film (10 $\mu \mathrm{m}$ thickness) gave a modulus of $2.936 \mathrm{GPa}(2 \mathrm{c}$, inset). 